\title{
Genetic Analysis of Patients with Chronic Active Hepatitis
}

\author{
Arthur R. Page, Harvey L. Sharp, Leonard J. Greenberg, and \\ EDMOND J. YUNIS \\ From the Departments of Pediatrics and Laboratory Medicine and Pathology, \\ University of Minnesota Medical School, Minneapolis, Minnesota 55455
}

\begin{abstract}
A B S T RACT 21 patients with chronic active hepatitis ( $\mathrm{CAH})$ and their families were HL-A typed. HL-A8 was significantly increased in frequency. An apparent increased frequency of HL-A1 was shown to be secondary to the increased HL-A 8 due to linkage disequilibrium. Genotype analysis revealed a striking increased frequency of homozygosity for HL-A8, 6 of 21 patients $(28.5 \%)$ vs. $2.8 \%$ of controls. Two patients and one normal who were homozygous for both HL-A1 and HL-A8 were found to be homozygous for a mixed lymphocyte culture (MLC) determinant 8a. Homozygous 8 a cells were used as test-stimulating cells in one-way MLC reactions to determine the frequency of the expression of the $8 \mathrm{a}$ determinant in 17 patients and 49 controls selected for HL-A type. $8 \mathrm{a}$ was found to be associated with $50 \%$ of HL-A8 haplotypes and was as frequent in the patient and control populations of the same HL-A types. These data suggest that susceptibility to $\mathrm{CAH}$ is determined by homozygosity for a gene that is in linkage disequilibrium with $\mathrm{HL}-\mathrm{A} 8$ and more closely associated with the HL-A second locus then with the locus for the major MLC determinant.
\end{abstract}

\section{INTRODUCTION}

An increased frequency of HL-A antigens 1 and 8 among patients with chronic active hepatitis was first reported by Mackay and Morris (1) and confirmed by Galbraith et al. (2). In this paper we report the results of HL-A genotyping of 21 chronic active hepatitis $(\mathrm{CAH})^{1}$ patients and an additional analysis of the fre-

Received for publication 17 April 1974 and in revised form 19 May 1975.

${ }^{1}$ Abbreviations used in this paper: $\mathrm{CAH}$, chronic active hepatitis; MLC, mixed lymphocyte culture; MLR-S, a locus. quency of an HL-A8-associated mixed lymphocyte culture (MLC) determinant, 8a, among patients and controls matched for HL-A types.

\section{PATIENTS AND METHODS}

The 21 patients in this study were diagnosed as having $\mathrm{CAH}$ by a clinical history of liver disease persisting longer than $3 \mathrm{mo}$, and liver biopsy showing the histology of chronic aggressive hepatitis (3) with or without cirrhosis. The patient group included 17 females and 4 males ranging in age from 10 to $38 \mathrm{yr}$. All patients received therapy with prednisone and/or 6-mercaptopurine for periods of 1 $20 \mathrm{yr}$. Eight patients were being treated at the time this study was done. As indicated by a normal serum ceruloplasmin and urinary copper excretion, none of these patients had Wilson's disease. It should be pointed out that this is a retrospective study done on surviving patients with $\mathrm{CAH}$ who, for the most part, have responded favorably to treatment. In our experience, about one-third of the patients with an initial diagnosis of $\mathrm{CAH}$ fail to respond to therapy and eventually die of this disease; we do not have any HL-A typing data on this latter group of patients.

The control group consisted of 216 healthy tumor-free Minnesota residents who were seen in the Cancer Detection Center Clinic at the University of Minnesota for an annual examination. All the controls and patients were of northern European heritage. The distribution frequency of HL-A antigens in this small control group does not differ significantly from the distribution frequency found by Morris, Metzger, Bluestone, and Terasaki (4) in a group of 1,863 normal white controls reported from Los Angeles.

$H L-A$ typing. HL-A antigens were determined with a standard two-stage dye exclusion microcytotoxicity test (5) using a panel of 90 well-characterized antisera capable of detecting all defined HL-A and most "W" specificities. HL-A haplotypes were assigned by conventional methods (6).

Differences in HL-A antigen frequency between patient and control groups were tested by $\chi^{2}$ analysis. The resulting $P$ values were not corrected for the number of antigen possibilities as is customary in surveys looking for abnormal frequency of any one of the many HL-A antigens. In this study it was not necessary to correct the 
resulting $P$ value for the number of HL-A antigens because we are only confirming previous reports that HL-A1 and $\mathrm{HL}-\mathrm{A} 8$ are increased in frequency.

$M L C$ assay. Unidirectional mixed culture reactivity was determined by a microculture technique described previously (7). The results were expressed as relative responses as described by J $\varnothing$ rgensen, Lamm, and Kissmeyer-Nielsen (8). The median response of each responding cell to four or five unrelated control-stimulating cells was set at $100 \%$. The relative response to the test-stimulating cells was calculated as follows:

Response to test cells (cpm)

Median response (cpm) $\frac{\text { - autologous control }(\mathrm{cpm})}{\mathrm{cpm})} \times 100$. - autologous control (cpm)

A responding cell that gave a relative response of less than $40 \%$, when stimulated by a test cell homozygous for the MLC determinant, was considered to carry that MLC determinant. The $40 \%$ value was chosen for two reasons: (a) J $\phi$ rgensen (8) found that family members who were known to carry a particularly MLC determinant could show relative responses of up to $40 \%$ when stimulated by cells homozygous for that determinant; $(b)$ In our experiments the relative responses of control cells, that did not share any HL-A antigens with the homozygous cells used as the test cells, ranged from 48 to $207 \%$ and appeared to represent a unimodal distribution.

TABLE I

Frequency of the HL-A Antigens in Patients with $C A H$

\begin{tabular}{|c|c|c|c|c|c|c|}
\hline \multicolumn{2}{|c|}{ HL-A } & \multicolumn{2}{|c|}{ CAH patients } & \multicolumn{2}{|c|}{$\begin{array}{c}216 \\
\text { Controls }\end{array}$} & \multirow[t]{2}{*}{$x^{2}$} \\
\hline & & $n$ & $\%$ & $n$ & $\%$ & \\
\hline \multicolumn{7}{|c|}{ First locus } \\
\hline $\mathrm{HL}$ & & 10 & 47.6 & 52 & 24.1 & $4.34^{*}$ \\
\hline “ & 2 & 11 & 52.5 & 129 & 59.7 & 0.18 \\
\hline “" & 3 & 1 & 4.8 & 57 & 26.4 & 3.7 \\
\hline “" & 9 & 0 & - & 42 & 19.4 & 3.7 \\
\hline “ & 10 & 5 & 23.8 & 33 & 15.3 & 0.5 \\
\hline “ & 11 & 3 & 14.3 & 22 & 10.2 & 0.04 \\
\hline W & 19 & 3 & 14.3 & 37 & 17.1 & 0.0007 \\
\hline “ & 28 & 1 & 4.8 & 14 & 6.5 & 0.02 \\
\hline \multicolumn{7}{|c|}{ Second locus } \\
\hline HL & & 1 & 4.8 & 27 & 12.5 & 0.48 \\
\hline & 7 & 2 & 9.5 & 56 & 25.9 & 1.97 \\
\hline “ & 8 & 13 & 61.9 & 39 & 18.1 & $19.0 \ddagger$ \\
\hline “ & 12 & 6 & 28.6 & 67 & 31.0 & 0.0002 \\
\hline “ & 13 & 1 & 4.8 & 13 & 6.0 & 0.06 \\
\hline $\mathrm{W}$ & 5 & 1 & 4.8 & 30 & 13.9 & 0.71 \\
\hline “ & 10 & 2 & 9.5 & 43 & 19.9 & 0.75 \\
\hline “ & 14 & 1 & 4.8 & 11 & 5.1 & 0.21 \\
\hline “ & 15 & 0 & - & 14 & 6.5 & 0.51 \\
\hline “ & 17 & 1 & 4.8 & 11 & 5.1 & 0.21 \\
\hline “ & 18 & 0 & - & 13 & 6.0 & 0.43 \\
\hline “ & 22 & 0 & - & 2 & 0.9 & 0.65 \\
\hline “ & 27 & 2 & 9.5 & 14 & 6.5 & 0.005 \\
\hline
\end{tabular}

* $P=0.05$.

$\ddagger P<0.001$.
TABLE II

Genotypes for 21 CAH Patients

\begin{tabular}{rrrc}
\hline $\begin{array}{c}\text { Patient } \\
\text { no. }\end{array}$ & \multicolumn{2}{c}{ Phenotype } & \multicolumn{2}{c}{ Genotype } \\
\hline 1 & 1,8 & $1-8$ & $1-8$ \\
2 & 1,8 & $1-8$ & $1-8$ \\
3 & 1,8 & $1-8$ & $1-8$ \\
4 & $1,2,8$ & $1-8$ & $2-8$ \\
5 & $1,2,8$ & $1-8$ & $2-8$ \\
6 & $1,10,8$ & $1-8$ & $10-8$ \\
7 & $1,8, \mathrm{~W} 27$ & $1-8$ & $1-\mathrm{W} 27$ \\
8 & $1,2,8, \mathrm{~W} 10$ & $1-8$ & $2-\mathrm{W} 10$ \\
9 & $2,11,8, \mathrm{~W} 5$ & $2-8$ & $11-\mathrm{W} 5$ \\
10 & $2,10,8,5$ & $2-8$ & $10-12$ \\
11 & $2,11,8,5$ & $11-8$ & $2-5$ \\
12 & $2,8,12$ & $\mathrm{X}-8$ & $2-12$ \\
13 & $\mathrm{~W} 19,8, \mathrm{~W} 14$ & $\mathrm{X}-8$ & $\mathrm{~W} 19-\mathrm{W} 14$ \\
14 & $2,10,12$ & $2-12$ & $10-12$ \\
15 & $2,11,12$ & $2-12$ & $11-12$ \\
16 & $2,10,12, \mathrm{~W} 17$ & $2-\mathrm{W} 17$ & $10-12$ \\
17 & $1,2, \mathrm{~W} 27$ & $2-\mathrm{W} 27$ & $1-\mathrm{X}$ \\
18 & $1,10,7,12$ & $1-7$ & $10-13$ \\
19 & $3, \mathrm{~W} 28,7, \mathrm{~W} 15$ & $3-7$ & $\mathrm{~W} 28-\mathrm{W} 15$ \\
20 & $\mathrm{~W} 19,12$ & $\mathrm{~W} 19-\mathrm{X}$ & $\mathrm{W} 19-12$ \\
21 & $\mathrm{~W} 19,10, \mathrm{~W} 10, \mathrm{~W} 15$ & $\mathrm{~W} 19-\mathrm{W} 10$ & $10-\mathrm{W} 15$ \\
\hline
\end{tabular}

$\mathrm{X}=$ Untypable antigen.

\section{RESULTS}

The frequency distribution analysis of HL-A antigens in patients and controls revealed that both HL-A1 and HL-A8 are increased in the patient group. HL-A1 was found in $48 \%$ of the patients vs. $24 \%$ of controls ( $P=$ $0.05)$, and HL-A8 was found in $62 \%$ of patients vs. $18 \%$ of controls $(P<0.001)$. None of the other antigen frequencies were significantly different between the two groups. See Table I.

By studying family members, we were able to assign genotypes for the HL-A antigens in the patient population (Table II). No anomalies in HL-A inheritance were found in any of the families. Six patients were homozygous for HL-A8 (28.5\%) and seven patients were heterozygous for $\mathrm{HL}-\mathrm{A} 8(33 \%)$, as compared to 2.8 and $15.3 \%$, respectively for homozygous and heterozygous HL-A8 in the control population. Four patients were homozygous HL-A1 $(19 \%)$ and six patients were heterozygous HL-A1 (28.6\%) compared to 0.9 and $23.1 \%$ homozygous and heterozygous HL-A1 in the control population. The control genotype frequencies are estimates assuming that a person with only one typable first or second locus antigen is homozygous for that antigen. This will overestimate the incidence of homozygosity because some people who are really heterozygotes with an untypable antigen at that locus will be included in the homozygous category. These re- 
TABLE III

Frequency of $H L-A$ Genotypes in 21 CAH Patients

\begin{tabular}{lccrccl}
\hline \multicolumn{1}{c}{ Genotype } & \multicolumn{2}{c}{ 21 patients } & \multicolumn{2}{c}{ 216 controls } & $x^{2}$ & \multicolumn{1}{c}{$P$} \\
\hline & $n$ & $\%$ & \multicolumn{1}{c}{$n$} & $\%$ & & \\
Homozygous 8 & 6 & 28.6 & 6 & 2.8 & 21.4 & $<0.0001$ \\
Heterozygous 8 & 7 & 33.3 & 33 & 15.3 & 3.25 & $>0.05$ \\
Homozygous 1 & 4 & 19.0 & 2 & 0.9 & 18.7 & $<0.0001$ \\
Heterozygous 1 & 6 & 28.6 & 50 & 23.1 & 0.08 & $>0.05$ \\
\hline
\end{tabular}

sults, summarized in Table III, indicate that most of the increased frequency of the HL-A1 and HL-A8 can be accounted for by the increased numbers of patients homozygous for these antigens.

We did not find any difference in severity of liver disease or associated vasculitis symptoms in the six patients who were homozygous for HL-A8 when compared to the other patients.

MLC studies were done between patients and controls selected for similar HL-A types. Stimulation in MLC has been shown to be a function of a locus (MLR-S)

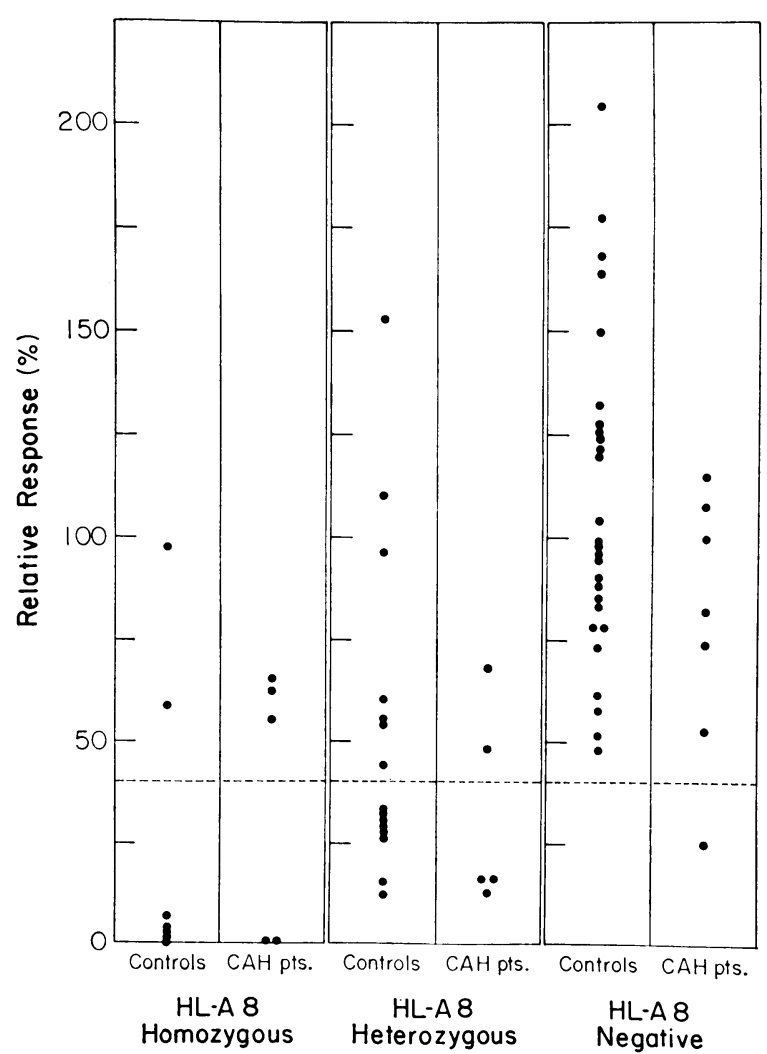

Figure 1 Relative responses of cells from $\mathrm{CAH}$ patients and normal controls to stimulation by test cells homozygous for both HL-A and MLR-S determinants Test cell genotype HL-A $(1-8) /(1-8)$ MLR-S $(8 a) /(8 a)$. Patients and controls are grouped according to HL-A type.
TABLE IV

Variability in the Relative Response to the Homozygous 8 a Test Cells on Different Days Using Different Control Cells to Determine the Median Response

\begin{tabular}{cccc}
\hline \multirow{2}{*}{$\begin{array}{c}\text { Responder } \\
\text { cells }\end{array}$} & \multicolumn{3}{c}{ Relative response, \% } \\
\cline { 2 - 4 } & Test 1 & Test 2 & Mean \\
\hline 1 & 0 & 1 & 0.5 \\
2 & 2 & 8 & 5.3 \\
3 & 0 & 0 & 0 \\
4 & 4 & 7 & 5.5 \\
5 & 28 & 32 & 30 \\
6 & 33 & 30 & 31.5 \\
7 & 32 & 43 & 37.5 \\
8 & 62 & 46 & 54 \\
9 & 168 & 103 & 135 \\
10 & 95 & 83 & 89 \\
11 & 60 & 119 & 89.5 \\
12 & 96 & 78 & 87 \\
13 & 153 & 89 & 121 \\
14 & 98 & 131 & 114.5 \\
\hline
\end{tabular}

that is closely associated with, but separable from, the second HL-A locus $(9,10)$. Recently, several groups of investigators have suggested the use of a stimulating cell known to be homozygous for MLR-S, to determine the frequency of that particular MLR-S allele in a population $(8,11-13)$. Lymphocytes from people who are either homozygous or heterozygous for the same MLR-S allele will respond poorly to the homozygous test cells. In contrast, heterozygous but not homozygous cells will stimulate homozygous cells.

In preliminary MLC studies between patients and controls selected for the same HL-A types, we found two patients and one control who apparently were homozygous for the same MLR-S allele. One of these patients was proven to be homozygous for MLR-S in a family study. We have named this allele MLR-S $8 \mathrm{a}$ because it is associated with HL-A8. This patient's cells were used as the stimulating cell to determine the frequency of MLR-S 8a in 17 of our own patients and in a population of 49 controls selected for HL-A type. The test genotype was HL-A (1-8)/(1-8) MLR-S (8a)/ (8a). We found that a large proportion of HL-A8-positive patients and controls were also carriers of MLR-S 8 .

5 of 7 HL-A 8 homozygous controls and 2 of 5 HL-A8 homozygous patients carry $8 \mathrm{a} ; 8$ of $15 \mathrm{HL}-\mathrm{A} 8$ heterozygous controls and 3 of $5 \mathrm{HL}-\mathrm{A} 8$ heterozygous patients carry 8a; none of $27 \mathrm{HL}-\mathrm{A} 8$-negative controls and 1 of 7 HL-A8-negative patients carry 8a. These results are summarized in Fig. 1. The proportion of patients carrying $8 \mathrm{a}$ was the same as the controls carrying $8 \mathrm{a}$ when the two groups were matched for HL-A type. 
The relative response values listed in Fig. 1 are calculated from the average of triplicate cultures done on 1 day. 14 of the 66 individuals were tested on a different day using the same homozygous 8a-stimulating cells but a different panel of control-stimulating cells to calculate the relative response. These repeat test values are listed in Table IV. For relative response values below $10 \%$, the variability is $\cong \pm 5 \%$. For relative response values between 20 and $60 \%$, the variability is $\cong \pm 10 \%$ and for relative response values about $60 \%$, the variability is $\cong+30 \%$. Only 1 of the 14 retests resulted in a value on the opposite side of $40 \%$. This was individual 7 , an HL-A8 heterozygote control, who had one test value of $32 \%$ and one of $43 \%$. We conclude from these results that single relative response determinations below 30 and above $50 \%$ are quite likely to be correctly assigned; however, there is uncertainty about the correct assignment to responder and nonresponder groups for single determinations that fall between 30 and $50 \%$. Only 6 of the 66 individual values fall into this range, and analysis of our results using a cutoff of either 30 or $50 \%$ as indicating nonresponse would not significantly alter the interpretation of our results.

\section{DISCUSSION}

This study of $21 \mathrm{CAH}$ patients confirms the previous reports $(1,2)$ of an increased frequency of HL-A1 and HL-A8 among patients with this disease.

Genotype analysis of the patient group revealed a striking incidence of homozygosity for $\mathrm{HL}-\mathrm{A} 1$ and HL-A8. Because the genes for HL-A1 and HL-A8 are known to occur together on the same chromosome much more frequently than would be expected for a random distribution of the two genes in the population, an increased frequency of one of these two antigens in the patient population might be due to this linkage disequilibrium of the two genes. If that is the case, one would expect that the association between the two antigens would be higher in the patient group than in the control group. Analysis of the data for linkage effect as suggested by Albert, Harms, Wank, SteinbauerRosenthal, and Scholz (14) revealed this to be the case. In the patient group, $80 \%$ of HL-A1 were associated with HL-A8, as compared to $42 \%$ in our control group, indicating that the increased frequency of $\mathrm{HL}-\mathrm{A1}$ is secondary to the frequency of HL-A 8 because of the linkage disequilibrium between the two genes. In contrast, the proportion of the HL-A8 coupled to HL-A1 was the same for the patient and control groups (54 vs. $56 \%$ ). Analysis of the frequency of HL-A1 and HL-A8 occurring without association with each other, shows that HL-A1 occurs without HL-A8 with the same frequency in the patient and the control population (14\%). However, HL-A8 occurs without HL-A1 with a higher frequency in the patient than in the control population ( 24 vs. $8 \%$ ). This analysis shows that the increased frequency of HL-A8 among the $\mathrm{CAH}$ patients is of primary importance, while the increased frequency of HL-A1 is a secondary effect due to the linkage disequilibrium between the two genes.

The MLC studies have identified an MLR-S allele, 8a, that shows a high degree of linkage disequilibrium with HL-A8. 8a was found in approximately $50 \%$ of persons heterozygous for HL-A8, but was found in only 1 of 34 HL-A8-negative persons. The frequency of MLR-S 8a was the same in the patient and control groups when matched for HL-A type. These results indicate that susceptibility to $\mathrm{CAH}$ is more closely associated with HL-A8 than with MLR-S 8a. This situation is clearly different from that described by Jersild et al. $(15,16)$. They identified an MLR-S allele, 7a, associated with HL-A7 and showed that patients with multiple sclerosis have a significantly higher frequency of $7 \mathrm{a}$ than HL-A genotype matched controls. Their studies indicate that multiple sclerosis susceptibility is more closely associated with MLR-S 7a than with HL-A7.

Three explanations for association between susceptibility to disease and histocompatibility antigens have been discussed by Snell (17) and McDevitt and Bodmer (18) : (a) The HL-A antigen of the cell surface might be a receptor site for virus attachment; (b) An infectious agent might contain antigens similar to the HL-A antigens and therefore, the host would be tolerant to these antigens. This is termed the "molecular mimicry" hypothesis; (c) The HL-A antigens themselves are not directly involved, but the genes responsible for susceptibility are in linkage disequilibrium with the HL-A genes. This last hypothesis is the most attractive of the three when trying to explain the need for homozygosity of an HL-A antigen. The first two explanations predict that the heterozygote would be susceptible while the third explanation is consistent with susceptibility associated with either the homozygous or heterozygous state.

The finding of an increased frequency of homozygosity for the HL-A 8 antigen in $\mathrm{CAH}$ patients is best explained if susceptibility to $\mathrm{CAH}$ is due to homozygosity for a gene associated with HL-A8. We can construct a model for susceptibility to $\mathrm{CAH}$ that is due to homozygosity for a gene that is in strong linkage disequilibrium with HL-A8. If this gene is associated five times more often with HL-A8 than with all other possible HL-A second-locus alleles, we can assign a frequency for the haplotype $\mathrm{HL}-\mathrm{A} 8 \mathrm{CAH}+=1$, and non-HL-A $8 \mathrm{CAH}+=0.2$. Then the relative frequency for homozygous $\mathrm{CAH}+$ can be calculated for homozygous HL-A8 $(1 \times 1=1)$; heterozygous HL-A8 $(1 \times$ 
$0.2=0.2) ;$ and non-HL-A8 $(0.2 \times 0.2=0.04)$. When these frequencies are multiplied by the frequency of HLA8 homozygous, heterozygous, and negative genotypes in the general population $(0.028,0.153,0.819)$ respectively, then a relative incidence for the distribution of HL-A8 genotypes associated with homozygosity for $\mathrm{CAH}+$ is $0.028,0.031,0.033$. Using this model we would expect $30 \%$ of $\mathrm{CAH}$ patients to be homozygous HL-A8, 34\% heterozygous HL-A8, and $36 \%$ non-HLA8. These expected values are in good agreement with the actual distribution of $28 \%$ homozygous HL-A8, $34 \%$ heterozygous $\mathrm{HL}-\mathrm{A} 8$, and $38 \%$ non-HL-A8 in our patient population.

It is interesting that the degree of genetic disequilibrium needed to make this model fit our data is similar to the degree of genetic disequilibrium between HL-A8 and MLR-S 8a. If the degree of genetic disequilibrium is an indirect measure of the distance between two genetic loci, then the distance between HL-A8 and MLR-S 8a should be about the same as the distance between HL-A8 and our postulated genetic locus for a gene governing susceptibility for $\mathrm{CAH}$. Since we have already established that $\mathrm{CAH}$ is more closely associated with the second HL-A locus than with the MLR-S locus, we are forced to conclude that the $\mathrm{CAH}$ susceptibility locus is within the HL-A region between the first and second HL-A loci.

Other investigators have reported finding an increased incidence of immunoglobulin abnormalities and an increased incidence of antibodies to mitochondria and smooth muscle in relatives of patients wtih $\mathrm{CAH}$ (19-22). Families with more than one documented case of $\mathrm{CAH}$ have also been reported $(20,21)$. These results could be explained either as a genetic propensity to produce autoantibodies or as a familial exposure to an infectious agent that carries antigens that crossreact with mitochondria or smooth muscle. It would be very helpful to do family studies measuring both HL-A genotypes and seroimmunologic parameters to determine linkage between a particular HL-A haplotype in a family and "auto"-antibody formation.

Several other diseases have been reported to be associated with an increased incidence of HL-A8. The most striking association is with coeliac disease (23, 24) and with Graves' disease (25).

It is interesting that Graves' disease and $\mathrm{CAH}$ have been found together in the same patients $(20,21,26,27)$ but we have not found any reports of an association between coeliac disease and $\mathrm{CAH}$.

Recently Albert et al. (14) did HL-A genotyping on 50 coeliac patients and did not find an increase in homozygosity for HL-A8. This suggests that coeliac disease may be due to a gene that causes susceptibility in the heterozygous state. The failure to find an asso- ciation between the two diseases might be explained if susceptibility to the two diseases is controlled by different allelic genes. Susceptibility to $\mathrm{CAH}$ would require homozygosity for the allelic pair and this would exclude the possibility of the susceptibility gene for coeliac disease occurring in the same person.

\section{ACKNOWLEDGMENTS}

This work was supported in part by U. S. Public Health Service grant PHS-5RO1-AI-10153-04 and grant RR-400 from general Clinical Research Center program, division of research resources, National Institutes of Health.

\section{REFERENCES}

1. Mackay, I. R., and P. J. Morris. 1972. Association of autoimmune active chronic hepatitis with HL-A1,8. Lancet. 2 : 793-795.

2 Galbraith, R. M., A. L. W. F. Eddleston, M. G. M. Smith, R. Williams, R. N. M. McSween, G. Watkinson, H. Dick, L. A. Kennedy, and J. R. Batchelor. 1974. Histocompatibility antigens in active chronic hepatitis and primary biliary cirrhosis. Br. Med. J. 3: 604-605.

3. De Grote, J., V. J. Desmet, P. Gedigk, G. Korb, H. Popper, H. Paulsen, P. J. Scheuer, M. Schmid, H. Thaler, E. Uehlinger, and W. Wepler. 1968. A classification of chronic hepatitis. Lancet. 2: 626-628.

4. Morris, R., A. L. Metzger, R. Bluestone, and P. I. Terasaki. 1974. HL-A W27-A clue to the diagnosis and pathogenesis of Reiter's Syndrome. N. Engl. J. Med. 290: 554-556.

5. Amos, D. B., W. Bashir, H. Boyle, M. MacQueen, and A. Tiilikainen. 1969. A simple micro cytotoxicity test. Transplantation (Baltimore). $7: 220-223$.

6. Amos, D. B. 1967. Determination of histocompatibility - the inheritance of leukocyte antigens. Transplantation (Baltimore). 5 : 1015-1023.

7. Greenberg, L. J., N. Reinsmoen, and E. J. Yunis. 1973. Dissociation of stimulation (MLR-S) and response (MLR-R) in mixed leukocyte culture by serum blocking factors. Transplantation (Baltimore). 16: 520-522.

8. Jørgensen, F., L. U. Lamm, and F. Kissmeyer-Nielsen. 1973. Mixed lymphocyte cultures with inbred individuals: an approach to MLC typing. Tissue Antigens. 3: $323-339$.

9. Yunis, E. J., and D. B. Amos. 1971. Three closely linked genetic systems relevant to transplantation. Proc. Natl. Acad. Sci. U. S. A. 68: 3031-3035.

10. Bach, F. H., M. B. Widmer, M. Segall, M. L. Bach, and J. Klein. 1972. Genetic and immunological complexity of major histocompatibility regions. Science (Wash. D. C.). 176: 1024-1027.

11. Mempel, W., H. Gross-Wilde, P. Baumann, B. Netzel, I. Steinbauer-Rosenthal, S. Scholz, J. Bertrams, and E. D. Albert. 1973. Population genetics of the MLC response: typing for MLC determinants using homozygous and heterozygous reference cells. Transplant. Proc. 5: 1529-1534.

12. van der Tweel, J. G., A. Blussé van Oud Alblas, J. J. Keuning, E. Goulmy, A. Termijtelen, M. L. Bach, and J. J. van Rood. 1973. Typing for MLC(LD): 1. lymphocytes form cousin-marriage offspring as typing cells. Transplant. Proc. 5: 1535-1538.

13. Dupont, B., C. Jersild, G. S., Hansen, L. S. Nielsen, M. Thomsen, and A. Svejgaard. 1973. Typing for 
MLC determinants by means of LD-homozygous and LD-heterozygous test cells. Transplant. Proc. 5: 15431549.

14. Albert, E. D., K. Harms, R. Wank, I. Steinbauer-Rosenthal, and S. Scholz. 1973. Segregation analysis of HL-A antigens and haplotypes in 50 families of patients with Coeliac Disease. Transplant. Proc. 5: 1785-1789.

15. Jersild, C., T. Fog, G. S. Hansen, M. Thomsen, A. Svejgaard, and B. Dupont. 1973. Histocompatibility determinants in multiple sclerosis with special reference to clinical course. Lancet. 2: 1221-1225.

16. Jersild, C., B. Dupont, T. Fog, G. S. Hansen, L. S. Nielsen, M. Thomsen, and A. Svejgaard. 1973. Histocompatibility-linked immune response determinants in multiple sclerosis. Transplant. Proc. 5: 1791-1796.

17. Snell, G. D. 1968. The H-2 locus of the mouse: observations and speculations concerning its comparative genetics and its polymorphism. Folia Biol. (Prague). $14: 335-358$.

18. McDevitt, H. O., and W. B. Bodmer. 1972. Histocompatibility antigens immune responsiveness and susceptibility to disease. Am. J. Med. 52: 1-8.

19. Cavell, B., and T. Leonhardt. 1965. Hereditary hypergammaglobulinemia and lupoid hepatitis. Acta Med. Scand. 177 : 751-759.

20. Elling, P., P. Ramlov, and P. Bildsoe. 1966. A genetic approach to the pathogenesis of hepatic cirrhosis. Acta
Med. Scand. 179: 527-533.

21. Galbraith, R. M., M. Smith, R. M. Mackenzie, D. E. Tee, D. Doniach, and R. Williams. 1974. High prevalence of seroimmunologic abnormalities in relatives of patients with active chronic hepatitis or primary biliary cirrhosis. N. Engl. J. Med. 290: 63-69.

22. Joske, R. A., and B. H. Laurence. 1970. Familial cirrhosis with autoimmune features and raised immunoglobulin levels. Gastroenterology. 59: 546-552.

23. Stokes, P. L., P. Asquith, G. K. T. Holmes, P. MackIntosh, and W. T. Cooke. 1972 Histocompatibility antigens associated with adult coeliac disease. Lancet. 2: 162-164.

21. Falchuk, Z. M., G. N. Rogentine, and W. Strober. 1972. Predominance of histocompatibility antigen HL-A 8 in patients with gluten-sensitive enteropathy. J. Clin. Invest. 51 : 1602-1605.

25. Grumet, C., J. Konishi, R. O. Payne, and J. P. Kriss. 1973. Association of Graves' disease with HL-A 8. Clin. Res. 21: 493. (Abstr.)

26. Page, A. R., R. A. Good, and B. Pollara. 1969. Long term results of therapy in patients with chronic liver disease associated with hypergammaglobulinemia. $\mathrm{Am}$. J. Med. 47 : 765-774.

27. Thompson, W. G., and I. R. Hart. 1973. Chronic active hepatitis and Graves' disease. Am. J. Dig. Dis. 18: 111-119. 